\section{Hollow-Shell Silicalite Nanostructures Formed on Sacrificial Latex Beads}

The zeolite silicalite has general applications in catalysis and separation processes. Recently, Sean Davis and coworkers at the School of Chemistry at the University of Bristol, U.K., and the MaxPlanck-Institute for Colloid and Surface Science in Golm, Germany, reported the assembly of silicalite nanoparticles into macroporous bulk materials. The assembly consisted of (1) the electrostatic deposition of multiple layers of nanoparticles onto polystyrene beads, and (2) the centrifugation of these heterostructures to obtain the bulk product. Calcination produced a silica material containing a hierarchical pore structure. Davis said, "[C]urrently at Bristol we are developing a number of strategies for the assembly (e.g. self-, directed-, templated) of nanoparticles into higher-order architectures. This particular assembly protocol has the advantage that it can be altered to control the pore diameter and wall thickness of the product."

As reported in the October 16 issue of Chemistry of Materials, silicalite nanoparticles were prepared by an established method using tetrapropylammonium hydroxide as the structure-directing agent. The nanoparticles had a mean diameter of $47 \pm 10 \mathrm{~nm}$, as measured by transmission electron microscopy.

The electrostatic deposition of the nanoparticles was accomplished by first coating the $640-\mathrm{nm}$ polystyrene beads with a layer of poly(diallyldimethylammonium chloride) (PDADMAC) to give the surface of the bead a positive charge. The modified beads were then exposed to the negatively charged nanoparticles. Subsequent nanoparticle layers were deposited by coating the last adsorbed layer with PDADMAC and again exposing the heterostructure to the nanoparticles. Up to five layers of nanoparticles were deposited on the beads. The average thickness of each layer was found to be $39 \mathrm{~nm}$.

The heterostructures were assembled into macroporous solids by centrifugation at $2000 \mathrm{rpm}$. This was followed by calcination above $800^{\circ} \mathrm{C}$ to remove the organic components and fuse the nanoparticles by silanol condensation. The product, characterized by transmission and scanning electron microscopies, was a macroporous solid having, on average, 500-nm-diameter pores, separated by 200 -nm-thick microporous walls for five nanoparticlelayer heterostructures, that is, a hollowshell, multilayer nanostructure. Electrondiffraction measurements showed that the walls separating the pores consisted of crystalline silicalite nanoparticles.

This approach to the production of bulk solids from nano-sized components differs from the usual method of introducing nanomaterials into an ordered superstructure lattice. The new method allows precise control over both pore size and wall thickness by controlling bead diameter and the number of capping layers, respectively. This method also allows for systematic modification of the wall composition by using heterostructures prepared with layers of several types of nanocrystals.

GREG KHITROV

\section{$\mathrm{C}_{60}$-Doped Zirconia Produces Homogeneous Dispersion of Carbon}

A multidisciplinary team of researchers in Japan is developing a procedure to obtain zirconia ceramics doped with $\mathrm{C}_{60}$.
Its main interest is to take advantage of the tribological properties of $\mathrm{C}_{60}$ while decreasing its high manufacturing costs. This combination is expected to result in a ceramic with outstanding mechanical properties and reduced friction resistance.

The scientists, from the University of Tokyo, the Electrotechnical Laboratory, and the National Institute for Research in Inorganic Materials, have obtained a uniform distribution of $\mathrm{C}_{60}$ in the zirconia matrix, as they report in the September issue of the Journal of the American Ceramics Society. This homogeneous distribution was achieved after mixing a combination of zirconium oxynitrate dihydrate (ZOD) and hexadecyltrimethylammonium chloride $\left(\mathrm{C}_{16}\right.$ TMA) in aqueous solution. $\mathrm{C}_{60}$ powders were added to obtain a final composition of $\mathrm{ZrO}_{2}-3$ mass $\% \mathrm{C}_{60}$. The powders were then dried and sintered in an ultrahigh-pressure machine at $5.5 \mathrm{GPa}$ and $600^{\circ} \mathrm{C}$ for $2 \mathrm{~h}$. The resulting density of

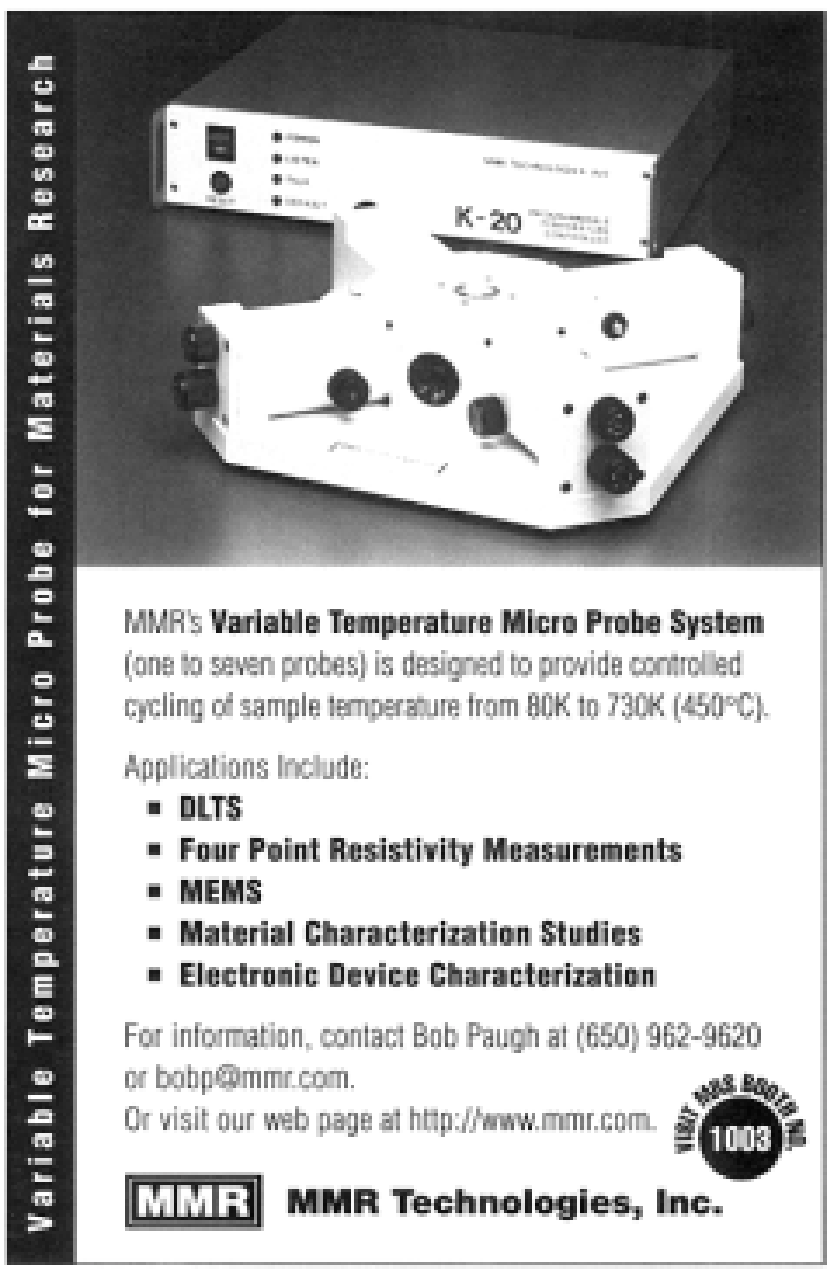

Circle No. 23 on Inside Back Cover 